\title{
Percepções Sobre Novembro Azul com Foco na Saúde Mental: Intervenção junto a uma Escola de Vigilantes
}

\author{
Ismael de Mendonça Azevedo ${ }^{I}$
}

\begin{abstract}
Resumo: O Objetivo deste trabalho é identificar o conhecimento do gênero masculino quanto ao movimento conhecido por Novembro Azul e despertar nos alunos de uma Escola de Vigilantes um diálogo de interesse sobre a promoção à saúde com foco específico na saúde mental no período deste movimento, dentro de uma perspectiva de promoção e bem estar integral ao gênero, diante de uma expectativa que vise propiciar a elaboração de conceitos quanto a Saúde Mental ao tempo que se propõe destacar o papel do Psicólogo no processo do cuidado em Saúde Mental. Para o alcance deste objetivo fora feita visita a Unidade Básica de Saúde para compreensão das dificuldades quando às demandas do gênero masculino e pesquisas quanto ao Novembro Azul e a saúde masculina nas bases Scielo e Google Acadêmico. De posse das pesquisas e da compreensão das demandas, elaborou-se uma dinâmica de grupo para aplicação junto aos alunos da Escola de Vigilantes, além de um momento expositivo sobre a ótica atual do Novembro Azul como perspectiva holística de cuidado à saúde, motivação que também insere a saúde mental. Com o estudo conclui-se que o conhecimento dos homens quanto ao Novembro azul está vinculado à percepção de que o câncer da próstata é o único fator deste movimento. Igualmente, há um desconhecimento geral quanto à visão da saúde mental como uma das formas de cuidado masculino a saúde.
\end{abstract}

Palavras-chave: Novembro Azul; Psicologia Organizacional; Saúde Mental.

\section{Perceptions about Blue November focusing on Mental Health: Intervention in a Watcher's School}

\begin{abstract}
The objective of this work is to identify the knowledge of the male gender about the movement known as Blue November and to awaken in the students of a School of Vigilantes a dialogue of interest about health promotion with specific focus on mental health during the period of this movement, within From a perspective of promotion and integral well-being to the gender, before an expectation that aims to propitiate the elaboration of concepts regarding Mental Health while it is proposed to highlight the role of the Psychologist in the process of care in Mental Health. In order to reach this objective, the Basic Health Unit was visited to understand the difficulties when it comes to the demands of the masculine gender and researches regarding the Blue November and the masculine health in the bases Scielo and Google Academic. With the research and the understanding of the demands, a group dynamics was developed for application to the students of the School of Vigilantes, as well as an expositive moment about the current perspective of the Blue November as a holistic perspective of health care, a motivation that also Inserts mental health. The study concludes that men's knowledge of blue November is linked to the perception that prostate cancer is the only factor in this movement. Likewise, there is general disregard for the view of mental health as one of the forms of male health care.
\end{abstract}

Keywords: November Blue; Organizational Psychology; Mental Health.

\footnotetext{
${ }^{1}$ Especialista MBA em Gestão de Pessoas pela Universidade Potiguar, Mossoró/RN, Brasil. Bacharel em Administração pela Universidade Estadual da Paraíba, Campina Grande/PB, Brasil. Discente do curso de Psicologia da Universidade Potiguar, Mossoró/RN. E-mail: ismaeldemendonca@bol.com.br
} 
Id on Line Revista Multidisciplinar e de Psicologia

Id on Line Multidisciplinary and Psycology Journal

\section{Introdução}

O homem e seus cuidados em saúde tem sido um objetivo de estudo instigante. Sua peculiar forma em não desviar uma especial atenção para os cuidados em saúde fez surgir um interesse no âmbito nacional de políticas voltadas para esse gênero. Por falta de tempo, receio ou machismo, sempre foi possível observar de maneira geral certo descuido da figura masculina quanto à prevenção e cuidado em saúde. Um paradigma se criou ao longo da história social masculina, o ser viril, provedor, mantenedor, não se permitiu ao longo de sua história se perceber como ser biológico capaz de padecer. A fragilidade esteve voltada sempre à figura feminina (KNAUTH; COUTO; FIGUEIREDO, 2012).

Com o nascimento e a influência das políticas públicas voltadas para o cuidado da saúde masculina é possível perceber um crescimento acerca da visão do homem e da sociedade em geral quanto a ações de promoção à saúde e prevenção de doenças do homem. Diante desta, a ampliação da publicização do Novembro Azul como um período especial de atenção integral a saúde masculina é de suma importância para a massificação da margem de homens que necessitam despertar um interesse em buscar também a atenção básica e primária à saúde. É necessário despertar um interesse preventivo em detrimento de sua atenção maior percebida ao uso do SUS como um sistema de saúde corretivo, reparativo e emergencial.

O fato é que em sua maioria os homens buscam o sistema de saúde por meio da atenção especializada, o que tem como consequência um agravamento e uma menor longevidade pelo retardo na atenção a doença. Muitos agravos poderiam ser evitados com a mudança na rotina e com medidas de prevenção primária, o que acarretaria menos sofrimento físico, emocional, psíquico, e, sobretudo familiar (BRASIL, 2009).

Nos últimos anos o serviço público de saúde tem buscado desenvolver projetos que atendam a demanda de homens em seu acolhimento. Trabalhos de publicização tem buscado desenvolver um sentimento de conscientização para que os homens busquem atendimento preventivo e rotineiro. O Novembro Azul é uma evolução do olhar estatal para a problemática masculina quanto à saúde.

$\mathrm{Na}$ contramão desse atendimento existe uma margem significativa de homens que desconhecem quais tipos de serviços estão dispostos aos mesmos nas ferramentas do Sistema 
Id on Line Revista Multidisciplinar e de Psicologia

Id on Line Multidisciplinary and Psycology Journal

Único de Saúde (SUS). Esse fator pode ser tido como um termômetro que pode medir o interesse do homem quanto aos cuidados pessoais em saúde.

Compreender qual o nível de conhecimento masculino sobre o Novembro Azul e enfatizar seu elo com a Saúde Mental deste gênero, é uma forma de avaliar o conhecimento que permeia o social, e, despertar nos homens uma proposta para a busca na geração de conhecimento sobre a importância da manutenção da sua saúde.

Entender como funciona cada aspecto que compreende tal pilar é uma forma de configurar de maneira adequada cada passo em busca de uma maior eficácia nos processos do Estado na publicização da saúde mental como parte da Saúde Pública. É necessário enfatizar que a saúde mental faz parte do Novembro Azul, para que esse pensamento se transforme em uma ação mental positivista, transformando a busca da saúde mental em um pilar de sustentação dos caminhos masculinos para plenitude de sua saúde.

Diante de tal cenário, esta intervenção se justifica especialmente pela complexidade que envolve o tema, além disso, auxilia na possibilidade de compreensão do imaginário masculino quanto ao Novembro Azul e seu link com a Saúde Mental. O Objetivo geral da intervenção visa despertar um diálogo de interesse sobre a promoção da efetiva atenção masculina à saúde com foco específico na saúde mental. Os objetivos específicos se articulam em: Identificar o conhecimento do público alvo quanto ao Novembro Azul, propiciar a elaboração de conceitos quanto a Saúde Mental e por fim destacar o papel do Psicólogo no processo de Saúde Mental.

\section{Percurso Histórico da PNAISH}

A história nos apresenta que homens sempre foram os formuladores e os executores de políticas públicas. Em sua maioria foram os principais idealizadores de programas que asseguraram privilégios de ordem simbólica e material a pelo menos alguns grupos de outros homens. As provocações, sobretudo feministas nas últimas décadas, implicaram no enquanto a necessidade da inclusão de gênero de maneira analítica e crítica para a implementação de avaliações e estratégia de políticas públicas e programas a fim de reduzir desigualdades. No âmbito internacional dois acordos foram determinantes para alimentar o discurso sobre a necessidade de enfocar o gênero masculino através de políticas públicas, sendo a Conferência 
Id on Line Revista Multidisciplinar e de Psicologia

Id on Line Multidisciplinary and Psycology Journal

Internacional sobre População e Desenvolvimento em 1994 no Cairo, e a IV Conferência Mundial sobre a Mulher em Pequim no ano de 1995 (LEAL; FIGUEIREDO; NOGUEIRA-DASILVA, 2012).

Especificamente no Brasil a Política Nacional de Atenção Integral à Saúde do Homem (PNAISH) nasce em grande parte do resultado obtido após deliberações sistemáticas que ocorreram entre abril e junho de 2008, quando foram promovidos cinco seminários com Sociedades Médicas, profissionais da saúde, pesquisadores, acadêmicos, representantes do CONASS e CONASEMS e de entidades civis organizadas para discutir os principais problemas de saúde que acometem os homens. A democratização veio em seguida, após as reuniões foi submetida à Consulta Pública para possibilitar a maior participação possível da sociedade. Diante da máxima fidelidade possível, um conjunto de ideias discutidas e pactuadas seguiu para os representantes dos setores responsáveis pela gestão e execução das ações. A institucionalização formal da Política deu-se ela Portaria 1.944 de 27 de agosto de 2009 (BRASIL, 2009).

É valido reforçar que mediante sua importância e aceitação social, as diretrizes da PNAISH de 2009 não são cristalizadas, inflexíveis. O Ministério da Saúde trabalha com a ressignificação em constantes movimentos de mudança quanto à proposição de reformulações na organização da abordagem da Área Temática de Saúde do Homem. Cabe aos gestores à prática constante de atualização do referencial teórico, ético e político, mediante constante diálogo com acadêmicos, profissionais e gestores em saúde. Essa perspectiva permite o rompimento com premissas unicamente biomédicas e medicalizantes, amplia horizontes para espaços onde se permite a influência histórica, críticas e contextualizadas de cada lugar do País (MARTINS; MALUMAT, 2013; COUTO; GOMES, 2012).

\section{(Des)Cuidado Masculino à Saúde}

A cultura masculina quanto aos cuidados em saúde é repleta de motivação, deveras superficial, para a não busca por serviços de saúde, sobretudo a busca ao Sistema Único de Saúde (SUS). No cotidiano masculino a saúde é vista como um plano que está abaixo das demandas de ocupações trabalhistas. Outras motivações quanto a sinais de fraqueza, 
Id on Line Revista Multidisciplinar e de Psicologia

Id on Line Multidisciplinary and Psycology Journal

impotência, medo e insegurança são apresentados como objeções, pois os homens não se sentem identificados com a fragilidade. Fragilidade que está intimamente identificada com imagens de crianças, mulheres e idosos. Preconceito e machismo que ainda estigmatizam entre a população masculina (SILVA; WAISSMANN, 2005; GOMES; NASCIMENTO; ARAÚJO, 2007; SILVA et al., 2010; ROCHA et al., 2016).

A imagem de que homem não adoece, somada ainda a comportamentos tidos como tipicamente masculinos como o uso de álcool, o tabagismo e a violência, anexos aos fatores culturais são tidos como os responsáveis por estes comportamentos que acabam por dificultar um acompanhamento mais holístico, incluindo ações de prevenção da população masculina. Atrelado a essa perspectiva ainda há um agravo dentro dos serviços quanto ao homem usuário, o de ser visto de maneira negativa por parte dos profissionais da assistência, pelo fato de apresentar pouca paciência na espera por atendimento (KNAUTH; COUTO; FIGUEIREDO, 2012).

\section{O Trabalho e as Principais Doenças Mentais}

O trabalho sempre propôs um ambiente repleto de paradoxos para o ser humano. Uma fonte de amor, prazer, ou ódio e dor. É correto afirmar que nos últimos anos as empresas vêm se tornando cada vez mais exigentes e duras, o mercado se torna cada dia mais hostil à saúde mental, pois se faz em meio a cobranças árduas de seus trabalhadores e, para isso, recorrem à gestão por meio de pressão (SOARES; VILLELA, 2012; AZEVEDO; AZEVEDO, 2016). Um funcionário dedicado, capacitado, competente, proativo, jamais ofereceria contribuições complacentes à organização se o estresse fosse sentido como o único resultado central da sua experiência de trabalho. O bem-estar do trabalhador deveria ser tão valorizado quanto o desempenho dos resultados capitais da organização. O estresse e a qualidade de vida no trabalho são fatores preponderantes, decorrentes do poder que os mesmos têm de impactar negativamente tanto nos indivíduos como nas organizações (AZEVEDO; AZEVEDO, 2016).

Os Transtornos Mentais e Comportamentais (TMC) fazem parte dos distúrbios referentes ao Capítulo V da CID-10, representam a terceira principal causa da concessão dos benefícios previdenciários caracterizado pelo auxílio-doença, com uma média anual de 9,3\% 
Id on Line Revista Multidisciplinar e de Psicologia

Id on Line Multidisciplinary and Psycology Journal

um montante de 34,9 para cada 10.000 segurados. As novas concessões aumentaram em média de 0,3\% ao ano. Os novos benefícios por TMC apresentam média anual de 203.299 casos. Os gastos impactam em uma média de 186 milhões de reais ao sistema previdenciário, com aumento de 7,1\% ao ano no valor com novos auxílios-doença por TMC. Os transtornos do humor, incluindo os quadros depressivos são os mais frequentes relacionados ao trabalho entre 2008 e 2010, e, o agrupamento dos transtornos neuróticos relacionados com o estresse e transtornos somatoformes assumiu a colocação do mais frequente motivo de auxílio-doença em 2011 (SILVA-JUNIOR; FISCHER, 2014).

\section{O Papel do Psicólogo}

Foram as necessidades trazidas pela prática de psicólogos nos serviços de saúde que estimularam as iniciativas de pesquisas desenvolvidas na psicologia voltadas para a saúde dos trabalhadores. Por não haver uma homogeneidade nas atividades laborais, não é possível destacar um respaldo teórico-conceitual homogêneo na perspectiva das teorias psicológicas que fundamentam os estudos. As demandas estão ligadas às necessidades sentidas no âmbito dos colaboradores em cada contexto (SATO; LACAZ; BERNARDO, 2006). A psicologia atuante no meio laboral, mais precisamente na saúde mental do trabalhador lhe abre um conjunto variado de possibilidades de atuação, como a consignação do nexo causal entre o trabalho e o adoecimento mental (JACQUES, 2007).

É principalmente como pesquisador social que o psicólogo participa da equipe multidisciplinar de vigilância, uma vez que pode atuar como um profissional sensível às formas particulares com que os trabalhadores veem os riscos de trabalho aos modos como eles lidam com os mesmos e como se organizam em cada microuniverso (BERNARDO, 2002; SATO, 1996; SATO; LACAZ; BERNARDO, 2006). O olhar do psicólogo também deve estar mais atendo aos fatores relacionados à organização dos processos de trabalho que podem ter consequências negativas para a saúde física e mental dos trabalhadores (SATO; LACAZ; BERNARDO, 2006). Cabe ao psicólogo avaliar as eminências de possíveis riscos a que os profissionais estão expostos. Em particular, pode efetivar-se em trabalhos preventivos à fadiga 
Id on Line Revista Multidisciplinar e de Psicologia

Id on Line Multidisciplinary and Psycology Journal

no trabalho ou ações que sejam remediadoras nos casos em que a síndrome já esteja instaurada (OLIVEIRA et al., 2010).

Quando das ações preventivas, o psicólogo pode analisar as situações de trabalho em relação ao modo de organização, ao ambiente físico, aos relacionamentos entre chefias e subordinados, entre outras, e propor, mudanças que sejam benéficas à saúde do trabalhador. Igualmente, o psicólogo pode exercer o papel de educador dentro da organização, seja mantendo diálogo permanente com a chefia a fim de apontar fatores organizacionais que possam propiciar o aparecimento da fadiga e mencionar as prováveis consequências para a própria organização, quanto promovendo palestras informativas, discussões e debates sobre o tema entre os trabalhadores (OLIVEIRA et al., 2010).

Em relação às ações remediadoras, é papel do profissional em psicologia inserido nas organizações o dever de orientar o trabalhador a procurar um perito em análise de doenças associadas ao trabalho ou recorrer ao Centro de Referência à Saúde do Trabalhador (CRST), onde é possível caracterizar a doença e fazer nexo casual do transtorno com o trabalho a partir de documentos específicos como a Comunicação de Acidente de Trabalho (CAT). Desta maneira o colaborador poderá exigir dos órgãos públicos benefícios como o auxílio-doença, o auxílio-acidente e pedidos de afastamento, assim como ser encaminhado para serviços públicos ou conveniados de saúde mental que ofereçam tratamento e apoio psicológico (OLIVEIRA et al., 2010).

As atividades inerentes ao psicólogo nas organizações ultrapassam os limites convencionais da área (LIMA; COSTA; YAMAMOTO, 2011), é dever deste profissional a constante atualização e busca de conhecimento sobre doenças relacionadas ao trabalho e seus sintomas, a fim de permitir embasar-se para um melhor acompanhamento e identificação de trabalhadores que estejam sofrendo de transtornos mentais provenientes de sua condição de trabalho bem como efetuar ou propor as devidas mudanças no ambiente laboral (OLIVEIRA et al., 2010).

\section{Metodologia}

O estudo se caracteriza como descritivo, por permiti a descrição das características da 
amostra e do fenômeno estudado, possibilitando estabelecer as relações (GIL, 2008), sendo um relato de experiência de abordagem qualitativa a partir de uma intervenção feita junto a uma escola de vigilantes no município de Mossoró/RN. A atividade teve como principal foco, uma palestra e uma dinâmica realizada com 21 alunos da referida escola, cuja temática discorreu sobre a percepção dos mesmos quanto ao cuidado do homem com a saúde mental como sendo uma das abordagens centrais da Política Nacional de Atenção Integral à Saúde do Homem.

Inicialmente dividiu-se a intervenção em dois momentos, sendo o primeiro um trabalho de reconhecimento dos saberes individuais. Buscou-se questionar os presentes sobre o conhecimento quanto à perspectiva do que seria o Novembro Azul. Durante o desenvolvimento da intervenção buscou-se evidenciar ainda quais fatores que os presentes vivenciam em sua realidade de vida, na expectativa de encontrar as motivações que os levam a perceber a influência em seu bem-estar psicológico.

O segundo momento se organizou como uma reflexão, tratando a perspectiva de trabalho como sendo uma parcela significativa da vida a ser explorada. Nesse contexto o trabalho se imprime como uma fonte de prazer e bem-estar ou como fonte de angústia e sofrimento. Foram expostas em sala as últimas pesquisas onde a saúde mental se apresenta como a $3^{\text {a }}$ maior causa de afastamento registrada (SILVA-JUNIOR, FISCHER, 2014).

O projeto se propôs a ser uma janela de pensamentos positivos na perspectiva de ir além de realizar intervenções em saúde de forma pontual, mas avaliar quais ações poderiam ser propostas na expectativa de incitar seu impacto na saúde mental do gênero abordado. As etapas da intervenção estão descritas no discorrer do trabalho. A construção do referencial decorreu durante o período de outubro a novembro de 2016, sendo necessário para tanto, o levantamento de referenciais teóricos e artigos científicos que embasassem a discussão e reflexão sobre a temática em periódicos científicos disponíveis nas bases SCIELO e GOOGLE ACADÊMICO.

\section{Resultados e Discussões}

Para a identificação das demandas e problemáticas foram estabelecidas conversações com profissionais atuantes na Unidade Básica de Saúde (UBS) com a finalidade de identificar 
Id on Line Revista Multidisciplinar e de Psicologia

Id on Line Multidisciplinary and Psycology Journal

as demandas oriundas da população local dentro da perspectiva de saúde do homem, iniciativa proposta dentro do Novembro Azul. Por meio do diálogo foi apontado que as ações do Novembro Azul existentes dentro do calendário anual são sempre possíveis de serem realizadas junto a todos os grupos de homens assistidos pela Unidade Básica de Saúde, porém, há ressalvas quanto a reduzida procura do público do gênero masculino ao serviço de saúde, sobretudo dentro de uma perspectiva de saúde mental. Diante dessa perspectiva, Gomes (2010), expressa que é essencial haver uma modificação na postura masculina frente à prevenção de doenças, de forma a superar questões estigmatizantes e mitos para ampliar a utilização dos serviços de saúde pelos homens.

O trabalho é uma atividade inerente ao indivíduo (OLIVEIRA et al., 2010). Diante desta, o público dos vigilantes constitui-se como de grande potencial para o desenvolvimento de ações voltadas ao Novembro Azul dentro de uma perspectiva de Saúde Mental. O nexo aporta-se na justificativa de que este grupo além da rotina, hábitos de vida e fatores genéticos que predispõem o surgimento de doenças, durante o desenvolvimento do trabalho estão expostos a uma série de possíveis tensões que podem agravar ou causar danos psicológicos, fatores capazes de interferir na sua condição de saúde mental e na qualidade de vida.

Frente à necessidade de levantar o conhecimento do grupo de vigilantes acerca da percepção e conhecimento sobre o que seria o Novembro Azul? Realizou-se tal questionamento ao grupo, no intuito de identificar a visão generalista do público sobre o tema. O grupo levantou a seguinte perspectiva: Novembro Azul é o mês do cuidado ao Câncer de Próstata (sic). Tal perspectiva remete a primeira abordagem de referência à saúde do homem no Brasil, quando no mês de novembro de 2008 o Instituto Lado a Lado pela Vida criou a campanha 'Um Toque, Um Drible', com o objetivo de promover mudanças no paradigma em relação à ida do homem ao médico (LAL, 2016). Assim, entende-se que a visão masculina quanto ao Novembro Azul não evoluiu no cotidiano do gênero, pois atualmente no referido mês se comemora um momento para os cuidados da saúde masculina de maneira holística.

Por passar grande parte da sua vida no ambiente de trabalho o homem está sujeito a diversos tipos de intercorrência que poderão repercutir negativa ou positivamente sobre sua saúde mental (OLIVEIRA et al., 2010). A partir desta, entendendo que o território laboral é vivo, dinâmico e histórico, no tocante ao planejamento das ações e dinâmica, a programação da atividade de intervenção destinada a proposta de uma visão sistêmica do Novembro Azul como 
um momento de cuidado a saúde masculina, incluindo a saúde mental, a atividade planejada teve o desígnio de propiciar ao grupo a aproximação entre conteúdos pertinentes à prevenção e a promoção da saúde mental, na medida que pôde ser exposto a todos o papel do psicólogo diante das demandas pertinentes a sua área no contexto discutido.

\section{Considerações Finais}

A intervenção contribuiu para elucidar equívocos do público presente quanto a atual exterioridade do Novembro Azul, além de atender algumas das necessidades de saúde promovendo o incentivo ao cuidado e prevenção. Tal momento serviu para proporcionar a inclusão da saúde mental enquanto necessidade de cuidado em função da sua dinâmica no ambiente de trabalho e sua complexa forma de influência. Foi possível semear o ideal no imaginário masculino que saúde mental não pode ser menosprezada ou abdicada, apresentando sua vasta relevância e efetividade na promoção da saúde do homem.

O estudo demonstra que há necessidade de divulgar o Novembro Azul como uma forma ampla de cuidado à saúde masculina, desvinculando sua ideologia em prol de um único fator, o câncer de próstata. É bastante eleger novas e eficazes formas de intervenção a fim de uma mudança eficiente dentro de uma perspectiva de cuidado holístico masculino em saúde. A promoção da saúde também se faz com o apoio de outros setores da sociedade, como escolas, por serem formadoras de opinião, e, desta forma fomentar o entendimento sobre a importância desse assunto, buscando uma saúde coletiva de nobreza.

Nessa perspectiva integradora, evidenciam-se as dificuldades de abordagens junto ao gênero masculino quanto a percepção de saúde mental como sendo parte integrante dos cuidados em saúde. É imprescindível ainda que novos pesquisadores voltem para estudos que sejam feitos junto ao público masculino como uma forma de elencar os estigmas que podem circular o imaginário dos homens. E a partir desse inventário, desenvolver novas formas e técnicas de abordagens dentro do movimento Novembro Azul para que essas pessoas despertem curiosidade e interesse de conhecimento em novas formas de cuidado em saúde, em especial em saúde mental. 
Id on Line Revista Multidisciplinar e de Psicologia

Id on Line Multidisciplinary and Psycology Journal

Com o estudo conclui-se que o conhecimento dos homens quanto ao Novembro azul está vinculado à percepção de que o câncer da próstata é o único fator deste movimento. Igualmente, há um desconhecimento geral quanto à visão da saúde mental como uma das formas de cuidado masculino a saúde.

\section{Referências}

AZEVEDO, I. M.; AZEVEDO, I. C. Assédio Moral: A Perversão Trazida Junto à Crise no Brasil. Um Relato De Experiência. In: Congresso de Stress da ISMA-BR, 16., 2016, Porto Alegre. Anais... Porto Alegre, 2016.

BERNARDO, M. H. Representações dos trabalhadores sobre os riscos em uma usina química: os acidentes e a contaminação nas representações dos trabalhadores. Cadernos de Psicologia Social do Trabalho, v. 5, p. 1-18, 2002.

Brasil. Ministério da Saúde (MS). Política Nacional de Atenção Integral à Saúde do Homem. Plano de Ação Nacional (2009-2011). Brasília: MS; 2009.

COUTO, M. T.; GOMES, R. Homens, saúde e políticas públicas: a equidade de gênero em questão. Ciência e Saúde Coletiva, v. 17, n. 10, p. 2569-2578, 2012.

GOMES, R. A saúde do homem em foco. São Paulo: Editora UNESP; 2010.

GOMES, R.; NASCIMENTO, E. F.; ARAUJO, F. C. Por que os homens buscam menos os serviços de saúde do que as mulheres? As explicações de homens com baixa escolaridade e homens com ensino superior. Cad. Saúde Pública, v. 23, n. 3, p. 565-574, 2007.

KNAUTH, D. R.; COUTO, M. T.; FIGUEIREDO, W. S. A visão dos profissionais sobre a presença e as demandas dos homens nos serviços de saúde: perspectivas para a análise da implantação da política nacional de atenção integral à saúde do homem. Ciência \& Saúde Coletiva, v. 17, n. 10, p. 2617-2626, 2012.

INSTITUTO LADO A LADO PELA VIDA (LAL). Disponível em: 〈http://www.ladoaladopelavida.org.br/campanha/novembro-azul >. Acesso em: 15 de nov. 2016.

LEAL, A. F.; FIGUEIREDO, W. S.; NOGUEIRA-DA-SILVA, G. S. O percurso da Política Nacional de Atenção Integral à Saúde dos Homens (PNAISH), desde a sua formulação até sua implementação nos serviços públicos locais de atenção à saúde. Ciência \& Saúde Coletiva, v. 17, n. 10, p. 2607-2616, 2012. 
LIMA, F. C.; COSTA, A. L. F.; YAMAMOTO, O. H. O exercício profissional do psicólogo do trabalho e das organizações: uma revisão da produção científica. Rev. Psi: Org e Trab R. Eletr. Psico, v. 11, n. 2, p. 21-35, 2011.

MARTINS, A. M.; MALAMUT, B. S. Análise do discurso da Política Nacional de Atenção Integral à Saúde do Homem. Saúde Soc, v. 22, n. 2, p. 429-440, 2013.

OLIVEIRA, J. R. S. et al. Fadiga no trabalho: como o psicólogo pode atuar? Psicologia em Estudo, v. 15, n. 3, p. 633-638, 2010.

ROCHA, E. M. et al. A política nacional de saúde do homem e os desafios de sua implementação na atenção primária à saúde. Revista Eletrônica Interdiciplinar, v. 1, n. 15, p. 43-48, 2016.

SATO, L. As implicações do conhecimento prático para a vigilância em saúde do trabalhador. Cadernos de Saúde Pública, v. 12, n. 4, p. 489-495, 1996.

SATO, L.; LACAZ, F. A. C.; BERNARDO, M. H. Psicologia e saúde do trabalhador: práticas e investigações na saúde pública de São Paulo. Estudos de Psicologia, v. 11, n. 3, p. 281-288, 2006.

SILVA-JUNIOR, J. S.; FISCHER, F. M. Adoecimento mental incapacitante: benefícios previdenciários no Brasil entre 2008-2011. Rev. Saúde Pública, v. 48, n. 1, p. 186-190, 2014.

SILVA, P. F.; WAISSMANN, W. Normatização, o Estado e a saúde: questões sobre a formalização do direito sanitário. Ciência e saúde coletiva, v. 10, n. 1, 2005.

SILVA, M. E. D. C. et al. Resistência do homem às ações de saúde: percepção de enfermeiras da estratégia saúde da família. Revista Eletrônica Interdisciplinar, v.3, n.3, p. 21-25, 2010.

SOARES, L. R.; VILLELA, W. V. O assédio moral na perspectiva de bancários. Rev. bras.saúde ocup, v. 37, n. 137, p. 203-212, 2012.

\section{Como citar este artigo (Formato ABNT):}

AZEVEDO, I.M. Percepções Sobre Novembro Azul com Foco na Saúde Mental: Intervenção junto a uma Escola de Vigilantes. Id on Line Revista Multidisciplinar e de Psicologia, Janeiro de 2017, vol.10, n.33, Supl 2. p.207218. ISSN: 1981-1179.

Recebido: 03/01/2017

Aceito: 17/01/2017 